\title{
Notes on the additions of Phaeophyceae from the coastline of Andhra Pradesh
}

\author{
Aron Santhosh Kumar $Y^{*}$ and Palanisamy $M$ \\ Botanical Survey of India, Southern Regional Centre, TNAU Campus \\ Coimbatore - 641 003, Tamil Nadu, India \\ *Corresponding author: santhosharon@gmail.com
}

\section{आंध्र प्रदेश के तटवर्तीय क्षेत्र में फियोफॉयसी शैवाल के अभिलेखन पर टिप्पणी अरोन संतोष कुमार वाई एवं पलनीसामी एम}

\section{सारांश}

आंध्र प्रदेश से फियोफॉयसी कुल के शैवालों पर महत्वपूर्ण वर्गिकी शोध पत्रों का या तो अभाव है या वे छिटपुट रूप में बिखरे पड़े हैं। विशाखापट्टनम जिले और तटवर्ती क्षेत्र में तो सर्वेक्षण किया जाना अभी भी शेष है। इसलिये प्रस्तुत शोध पत्र में फियोफॉयसी कुल की प्राप्ति हेतु मार्च 2017 से अगस्त 2019 तक आंध्र प्रदेश के तटवर्ती जिलों के 112 क्षेत्रों में सर्वेक्षण कार्य सम्पादित किया गया। सर्वेक्षणोपरान्त 4 गणों की 27 जातियां जो 12 वंशों और 6 कुलों से सम्बन्धित हैं को सूचीबद्ध किया गया है जिनकें 15 जातियों का आंध्र प्रदेश के शैवाल वनस्पतिजात के लिये नवीन वितरणपरक अभिलेख के तौर पर दर्ज किया गया है। प्रस्तुत शोधपत्र में प्रत्येक जाति का संक्षिप्त वर्णन, प्राप्ति समय, निरिक्षित प्रतिरूप के साथ दिया गया है।

\section{ABSTRACT}

The crucial floristic reports of the members of Phaeophyceae (Brown seaweeds) from Andhra Pradesh are sporadic, inadequate and merely reported from Visakhapatnam district and remaining coastal areas are yet to be explored. Hence, the present attempt was engaged to investigate the occurrence of Phaeophyceae from 112 stations of coastal districts of Andhra Pradesh between March 2017 and August 2019. The current endeavours revealed occurrence of 27 species belonging to 12 genera of 6 families under 4 orders, out of which 15 species are reported as new distributional records to the algal flora of Andhra Pradesh. Each taxon is appended here with a brief description, time of occurrence and specimens examined.

Keywords: Andhra Pradesh, Brown Seaweeds, New records, Visakhapatnam

\section{INTRODUCTION}

Phaeophyceae (Brown algae) is the peculiar class with specialized characters and reside in the natural environment. The organization of the plant body ranges from unicellular to multicellular with a great level of morpho-plasticity and they can attain a length of 60-150 meters. Members of this class recognised for their ubiquitous distribution in a wide range.
Habitually, they dwell in the marine ecosystem, rarely evidenced by freshwater bodies (Rott \& Wehr, 2016). The colour of the algae is characterized by the pigments chlorophyll a (Chl a), chlorophyll c (Chl c) and xanthophyll. Alcohol, fats, laminarin and fucoidan are the reserve food material (Gunwant Sokhi \& Vijayaraghavan, 1986). Reproduction of this algal group befalls of asexual (motile \& non-motile spores) and sexual way (isogamy, anisogamy to oogamy). 
It represents 65 families under 25 orders with 2058 taxa worldwide and in India, 211 taxa of 50 genera belong to 13 families were reported by several pioneer workers (Rao \& Gupta, 2015).

The evidence on the occurrence and distribution of brown algae on the east coast especially of Andhra Pradesh is limited and poorly swotted by a few of the researchers. Rao \& Sreeramulu, (1970a) reported 16 species of Phaeophyceae from the coastline of Visakhapatnam for the first time. Whilst, Sreeramulu (1952 \& 1953); Rao \& Sreeramulu (1964); Rao \& Sreeramulu, (1968); Rao \& Rao (1986); Rao \& al. (2008); Prasanna Lakshmi \& Narasimha Rao (2009); Satya Rao \& al. (2011); Narashimha Rao \& Srinivasa Rao, (2016); Sowjanya \& Sekhar (2015 \& 2017) were inspected the marine macroalgal diversity of Andhra Pradesh and merely focused on the coastline of Visakhapatnam. Information on the class Phaeophyceae from the northern (Srikakulam and Vizianagaram) and southern coastline (East Godavari, West Godavari, Krishna, Guntur, Prakasam, and Nellore) of Andhra Pradesh are inattentive. In this view, this present study is subjected to provide a detailed account of Phaeophyceae of Andhra Pradesh.

\section{MATERIALS AND METHODS}

Andhra Pradesh is geographically positioned between $12^{\circ} 41^{\prime}-19^{\circ} 07^{\prime} \mathrm{N}$ latitude and $69^{\circ} 37^{\prime}-84^{\circ} 44^{\prime} \mathrm{E}$ longitude in the southern part of the subcontinent. The boundaries of the state are restricted by Tamil Nadu to the south, Karnataka to the southwest and west, Telangana to the northwest and north, and Odisha to the northeast. The eastern part of the state is bounded by the coastline along the Bay of Bengal. Among the other coastal states, Andhra Pradesh has got a coastline of around c.973 km, running from Pulicat Lake (Nellore district) in the south to Donkuru (Srikakulam District) in the North. The offshore rocky plateaus stretched from Srikakulam district to Visakhapatnam district with an enormous diversity of seaweeds and the remaining coastline is endowed with the sand beach.

(Plate 1)

A sum of 112 localities was selected for the collection of marine macro algae during low tides in different seasons from March 2017 - August 2019. The collected samples were preserved using standard methodologies of Wet Preservation (Liquid preservation) and Dry Preservation (Herbarium). The identification of the seaweeds was done by morphological \& microscopic observations, referring standard books, literatures \& articles. Further, consulted the herbarium specimens deposited at MH, Coimbatore, ISIM, Kolkata and CSIR-CSMCRI-MARS, Ramnad. The nomenclature uncertainties were diagnosed with the aid of online resources to provide the standard data on the Phaeophycean of Andhra Pradesh.

\section{RESULTS}

The present survey reveals the occurrence of 27 species of phaeophyceae belong to 12 genera of 6 families under 4 orders. Among them, Dictyotales is the predominant order and encompasses 06 genera (Dictyopteris, Dictyota, Padina, Lobophora and Stoechospermum) of Dictyotaceae with 11 species. The order Fucales represents the family Sargassaceae with 9 species of the genera Sargassum (8 species) and Turbinaria (1 species). Ectocarpales is the moderated order, signifies with 6 species belong to 4 genera (Ectocarpus, Chnoospora, Rosenvingea and Feldmannia) under the families Ectocarpaceae (1 species) and Scytosiphonaceae (3 species) Acinetosporaceae (2 species). Sphacelariales is the least order includes the family Scytosiphonaceae with one taxon.

Also, the findings of the present work reveals the new distributional additions (15 species) namely Ectocarpus siliculosus, Chnoospora bicanaliculata, Rosenvingea intricate, Dictyopteris australis, Dictyota ciliolata, Padina australis, Padina boergesenii, Padina boryana, Padina gymnospora, Padina pavonica, Stoechospermum polypodioides, Sargassum cinctum, Sargassum cinereum, Sargassum swartzii and Turbinaria conoides to the marine macro algal flora of Andhra Pradesh, East Coast of India.

Ectocarpus, Chnoospora, Dictyota, Padina and Sargassum are the mutual genera reported with the regular periodical interval in all season. Rosenvingea, Feldmannia, Sphacelaria and Lobophora are seasonal genera collected poorly in Andhra Pradesh during monsoon.

The genera Dictyopteris, Stoechospermum \& Turbinaria are reported for the first time on this 

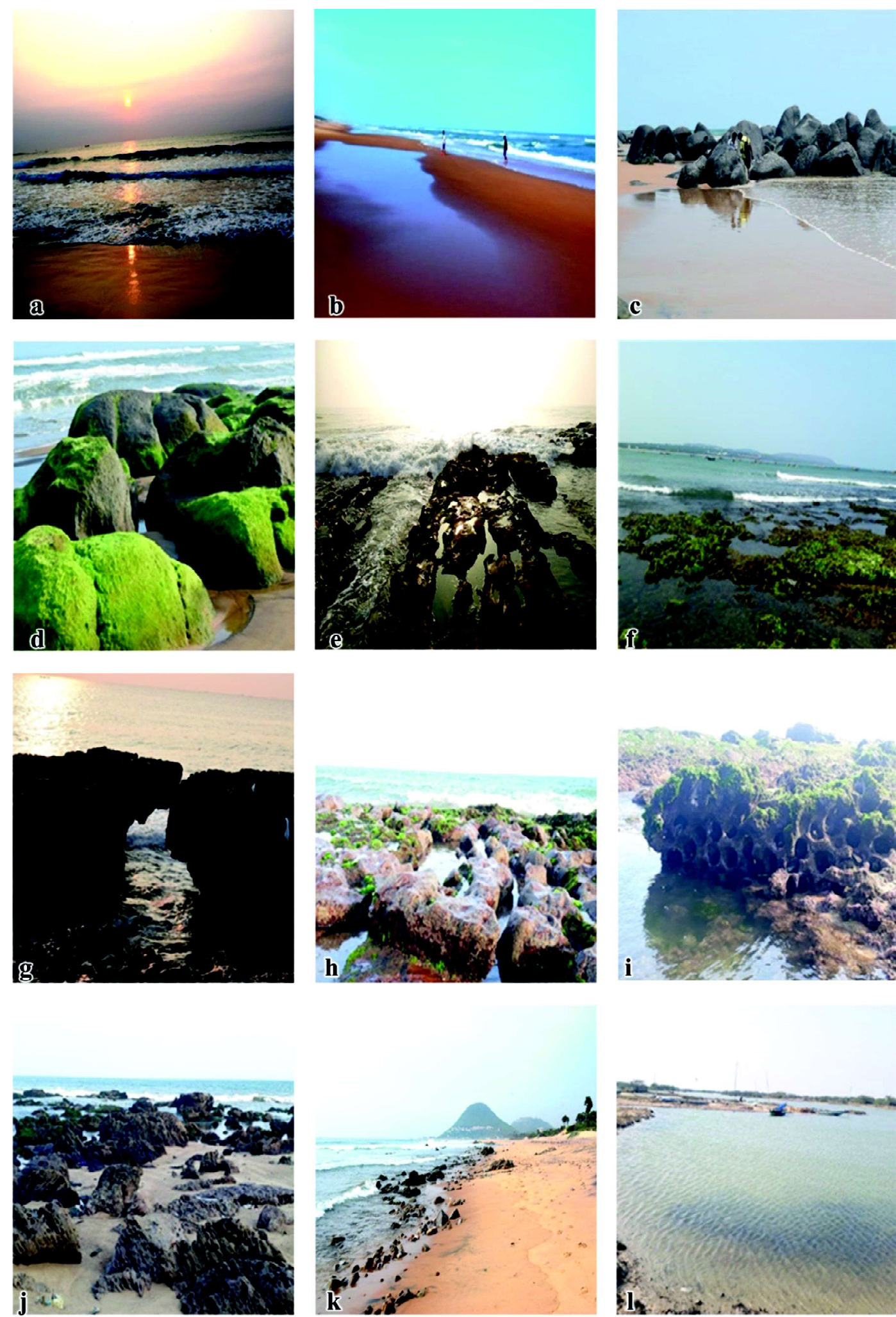

Plate 1: Different coastal types of Andhra Pradesh: a. Donkuru; b. Baruva; c. Yeramukkam; d. Mugadalapadu; e. Bheemlipatnam; f. Mangamaripeta; g. Thotlakonda; h. Thimmapuram; i. Guduvanipeta; j. R.K. Beach; k. Rushikonda; l. Pulicat Lake. 
coastline. The investigation of Sowjanya \& Sekhar (2015) clearly validates the absence of 41 species when compared to data of 1964 - 1970 obtained from this coast of Andhra Pradesh by former investigators; among them 12 species are phaeophycean. The present study highlights the growth of Sphacelaria tribuloides, Feldmannia irregularis and Feldmannia mitchelliae after the foremost investigation by Rao \& Sreeramulu (1970a). Subsequently, all the 27 species have been arranged systematically and enumerated with description, occurrence, species examined and distribution.

\section{Order: Ectocarpales; Family: Ectocarpaceae}

Ectocarpus siliculosus (Dillwyn) Lyngb., Tent. Hydrophytol. Dan.: 131, figs. 43B, C. 1819. Basionym: Conferva siliculosa Dillwyn.

Plant body olive green to light brown in colour, filamentous, 3-5 cm long, caespitose, heterotrichous. Epilithic, rarely epiphytic, holdfast minute, rhizoidal or discoidal. Profusely branched to form a dense tufted upright plant body. Cells, thin walled, filamentous, uniseriate, rectangular to cylindrical, $6-15 \times 4-12 \mu \mathrm{m}$ in size. Centrally located reticulate chloroplast with several pyrenoids. Sporangia lateral, plurilocular, sessile, elongated with obtuse apex.

Specimen examined: Andhra Pradesh: Visakhapatnam District- Bheemunipatnam coast, 09.09.2018, Palanisamy \& Aron Santhosh Kumar 137253 (MH).

Season: Monsoon; Distribution: Gujarat, Karnataka, Kerala \& Tamil Nadu.

\section{Family: Scytosiphonaceae}

Chnoospora bicanaliculata V. Krishnam. \& P.C. Thomas in Seaweed Res. Utiln. 2:44.1977. (Plate 2: a)

Thallus golden brown to dark brown in colour, 4-13 cm long, cylindrical to terete, tufted, forked, epilithic with discoidal holdfast. Stipe stalked, $1-7 \mathrm{~cm}$ long and fronds sub-cylindrical to slightly flattened. Subdichotomously branched with two distinct grooves on both sides. Margins entire, apex acute to obtuse. Cells in surface view elongate-rectangular, $4-6 \times 2-4.5 \mu \mathrm{m}$, multi layered, cells range from $45-65 \mu \mathrm{m}$ thickness. Reproductive bodies embedded on frond surface, sporangia plurilocular, growing in sori.

Specimen examined: Andhra Pradesh: Srikakulam
District- Bandaruvanipeta, 07.09.2018, Palanisamy \& Aron Santhosh Kumar 137538 (MH).

Occurrence: Monsoon; Distribution: Laccadive Islands \& Tamil Nadu.

Chnoospora minima (K. Hering) Papenf. in J. S. African Bot. 22:69. 1956; Basionym: Fucus minimus Hering.

(Plate 2: b)

Dark brown in colour, cylindrical, $4-9 \mathrm{~cm}$ long, rigid, tufted, epilithic. Fronds sub cylindrical, wiry, $4-6 \mathrm{~cm}$ long and dichotomously branched, slightly compressed with sunken points at dichotomy, margins entire, acute to rounded apex. Surface view of the cells rectangular or elongated, meristematic, multi-layered and thick walled. Reproductive bodies at the surface of the thallus, sporangia saclike, plurilocular.

Specimen examined: Andhra Pradesh: Srikakulam District- Laxmipuram, 21.08.2019, Palanisamy \& Aron Santhosh Kumar 140379 (MH).

Occurrence: Monsoon; Distribution: Tamil Nadu \& Kerala.

Rosenvingea intricata (J.Agardh) Boergesen Dansk Bot. Ark. 2.2: 26, 1914. Basionym: Asperococcus intricatus J.Agardh.

(Plate 2: c)

Thallus yellowish-brown to pale brown in color, usually 3-6 cm long, erect, tubular or slightly flattened, tufted. Epilithic, holdfast small, rhizoidal, stipe stalked, tubular, up to $0.5 \mathrm{~cm}$ long. Fronds irregularly or dichotomously branched; older branches sometimes produce short laterals, unequal, slightly curved; margins entire, intricated projection at apical zone and apex or obtuse. Bundles of hairs scattered over surfaces. Plurilocular sporangia developing from epidermal cells, in sori.

Specimen examined: Andhra Pradesh: Nellore DistrictPulicat, 27.03.2017, Palanisamy \& Aron Santhosh Kumar 137257 (MH).

Occurrence: Post Monsoon; Distribution: Gujarat and Tamil Nadu.

\section{Family: Acinetosporaceae}

Feldmannia mitchelliae (Harv.) H.-S.Kim Algal flora of Korea. 2(1): 51.2010; Basionym: Ectocarpus mitchelliae Harv.

Thallus olive green to dark brown in color, filamentous, 4-7 cm long, filamentous, heterotrichous, densely tufted, usually epilithic. Chromatophore 

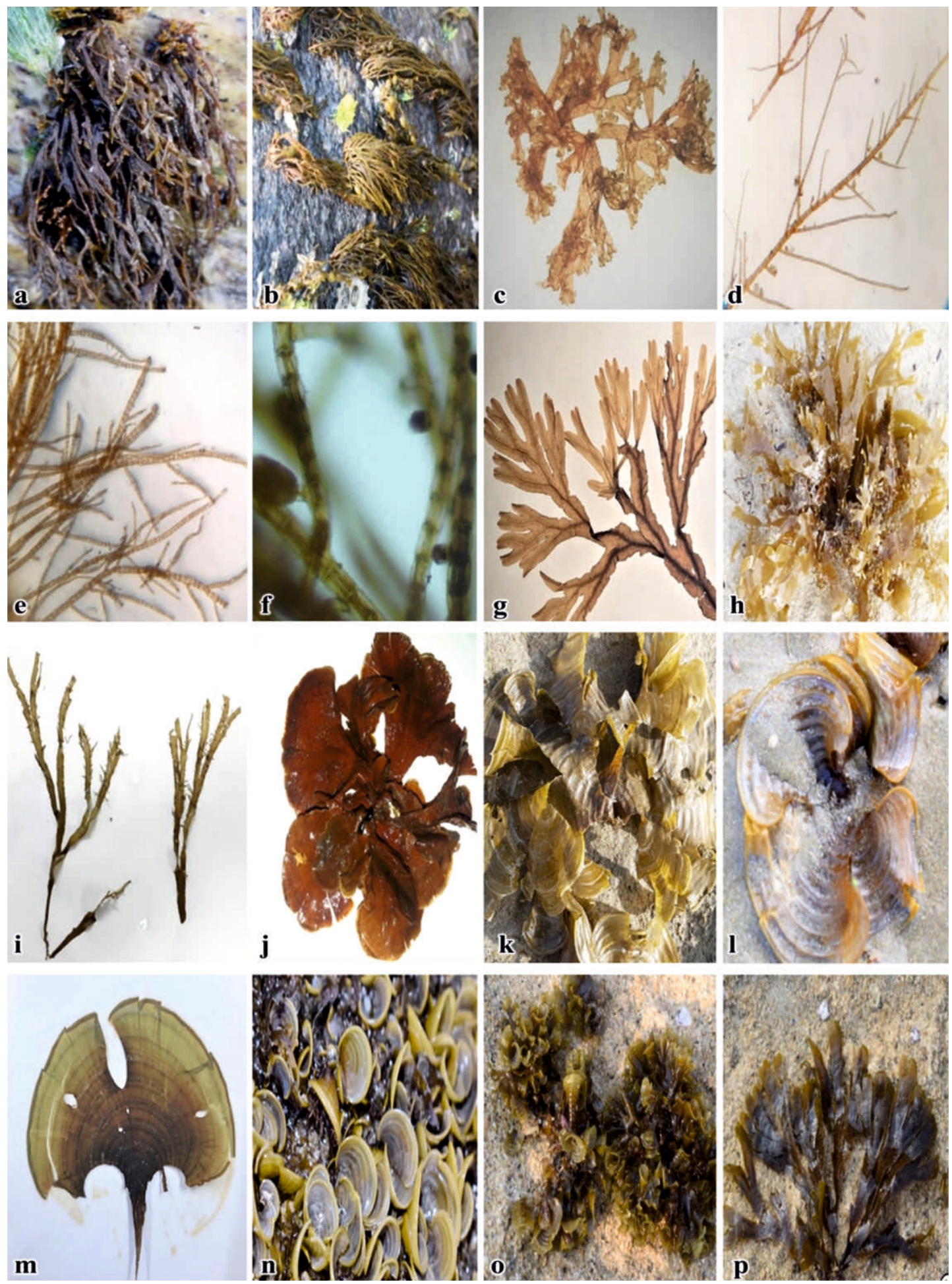

Plate 2: Habits of Phaeophyceae: a. Chnoospora bicanaliculata; b. Chnoospora minima; c. Rosenvingea intricata; d. Feldmannia irregularis; e. \& f. Sphacelaria tribuloides with plurangia; g. Dictyopteris australis; h. Dictyota dichotoma; i. Dictyota ciliolata; j. Lobophora variegata; k. Padina boergesenii; 1 . Padina boryana; m. Padina gymnospora; n. Padina pavonica; o. Padina tetrastromatica; p. Stoechospermum polypodioides. 
numerous, discoidal. Sporangia plurilocular.

Specimen examined: Andhra Pradesh: Visakhapatnam District- Bheemunipatnam, 21.03.2019, Palanisamy \& Aron Santhosh Kumar 138068 (MH).

Occurrence: Usually post-monsoon season; Distribution: Goa, Maharashtra and Tamil Nadu.

Feldmannia irregularis (Kuetz.) Hamel, Pheophyc. France 5: pl. 17. 1937; Basionym: Ectocarpus irregularis Kuetz.

(Plate 2: d)

Thallus light-dark brown in color, filamentous, large, up to $8 \mathrm{~cm}$ long, tufted, usually develop as epiphyte on other algae. Filamentous, heterotrichous, pseudo dichotomously or irregularly branched and gradually tapering towards apex. Cells cylindrical and having numerous chromatophore, discoidal.

Specimen examined: Andhra Pradesh: Visakhapatnam District- Mangamaripeta, 21.03.2019, Palanisamy \& Aron Santhosh Kumar 140304 (MH).

Occurrence: Usually post-monsoon season; Distribution: Maharashtra and Tamil Nadu.

\section{Order: Sphacelariales;}

\section{Family: Sphacelariaceae}

Sphacelaria tribuloides Meneghini, Lett. Corinaldi a Pisa (2):2.1820.

(Plate 2: e \& f)

Plant body golden brown in colour, growing up to 2.5 $\mathrm{cm}$ long. epiphytic, forming dense tufts. Filaments erect, irregulary branched, $25-55 \mu \mathrm{m}$ broad, and segments $20-45 \mu \mathrm{m}$ long. Propagules broad, cordate or triangular, and stalked. Sporangia globose, with small stalk and plurangia cylindrical,sub- sessile or with short stalk.

Specimen examined: Andhra Pradesh: Srikakulam District-Akkupalli, 22.09.2017, Palanisamy \& Aron Santhosh Kumar 137298 (MH).

Occurrence: Monsoon season; Distribution: Andaman \& Nicobar, Gujarat and Tamil Nadu.

\section{Order: Dictyotales; Family: Dictyotaceae}

Dictyopteris australis (Sond.) Askenasy, Forsch. Botanik: 30. 1888. Basionym: Haliseris australis Sonder

(Plate 2: g)

Thallus light to dark brown in color, flattened, up to 8 $\mathrm{cm}$ long, tufted with discoidal Stipe slightly slender, small, up to $0.8 \mathrm{~cm}$ long. Fronds dichotomous delicate, surface, midrib prominent, margins entire to slightly wavy and apex rounded to obtuse. Thallus multilayered, 4-6- layered, small, cells squarish to rectangular. Reproductive scattered at surfaces of the fronds.

Specimen examined: Andhra Pradesh: Visakhapatnam District- Thimmapuram, 23.03.2017, Palanisamy \& Aron Santhosh Kumar 137207 (MH).

Occurrence: Post monsoon; Distribution: Gujarat, Goa, Karnataka, Kerala and Tamil Nadu.

Dictyota dichotoma (Huds.) J.V. Lamour. in J. Bot. (Desvaux) 2: 42. 1809. Basionym: Ulva dichotoma Hudson.

(Plate 2: h)

Thallus dark-yellowish brown in color, usually $2-8 \mathrm{~cm}$ long, leafy to frondose or ribbon like, bushy, tufted, epilithic. Holdfast discoidal, firmly attached substrata Fronds foliose, up to $8 \mathrm{~cm}$ long, membranous, regularly dichotomously branched; branches profuse towards apex, flat, uniform, slightly broader at base; margins entire. Cells in surface view squarish, thick walled, compact, chromatophore content less. Sporangia develop in sori on surface.

Specimen examined: Andhra Pradesh: Visakhapatnam District- Mangamaripeta, 10.09.2018, Palanisamy \& Aron Santhosh Kumar 137862 (MH).

Occurrence: Post monsoon; Distribution: Andaman \& Nicobar, Goa, Gujarat, Karnataka, Kerala and Tamil Nadu.

Dictyota ciliolata Sonder ex Kuetz., Tab. Phycol. 9: 27. 1859.

(Plate 2: i)

Thallus golden yellow to light brown in color, flattened, 4-12 cm long, and 0.4-1.2 cm wide. Leafy, ribbon like, bushy, tufted with discoidal holdfast. Fronds foliose, 3$7 \mathrm{~cm}$ long, membranous, regularly dichotomously branched, margins ciliate to irregularly dentate or proliferated towards apex. Cells in surface rectangular to polygonal, thick walled, hyaline chromatophore contents less. Sporangia develop on both surfaces of the fronds, round to spherical in shape.

Specimen examined: Andhra Pradesh: Visakhapatnam District- Guduvanipallem, 22.03.2019, Palanisamy \& Aron Santhosh Kumar 140350 (MH).

Occurrence: Post monsoon; Distribution: Andaman \& Nicobar, Goa, Gujarat, Karnataka, Kerala and Tamil Nadu.

Lobophora variegata (J.V. Lamour.) Womersley ex 

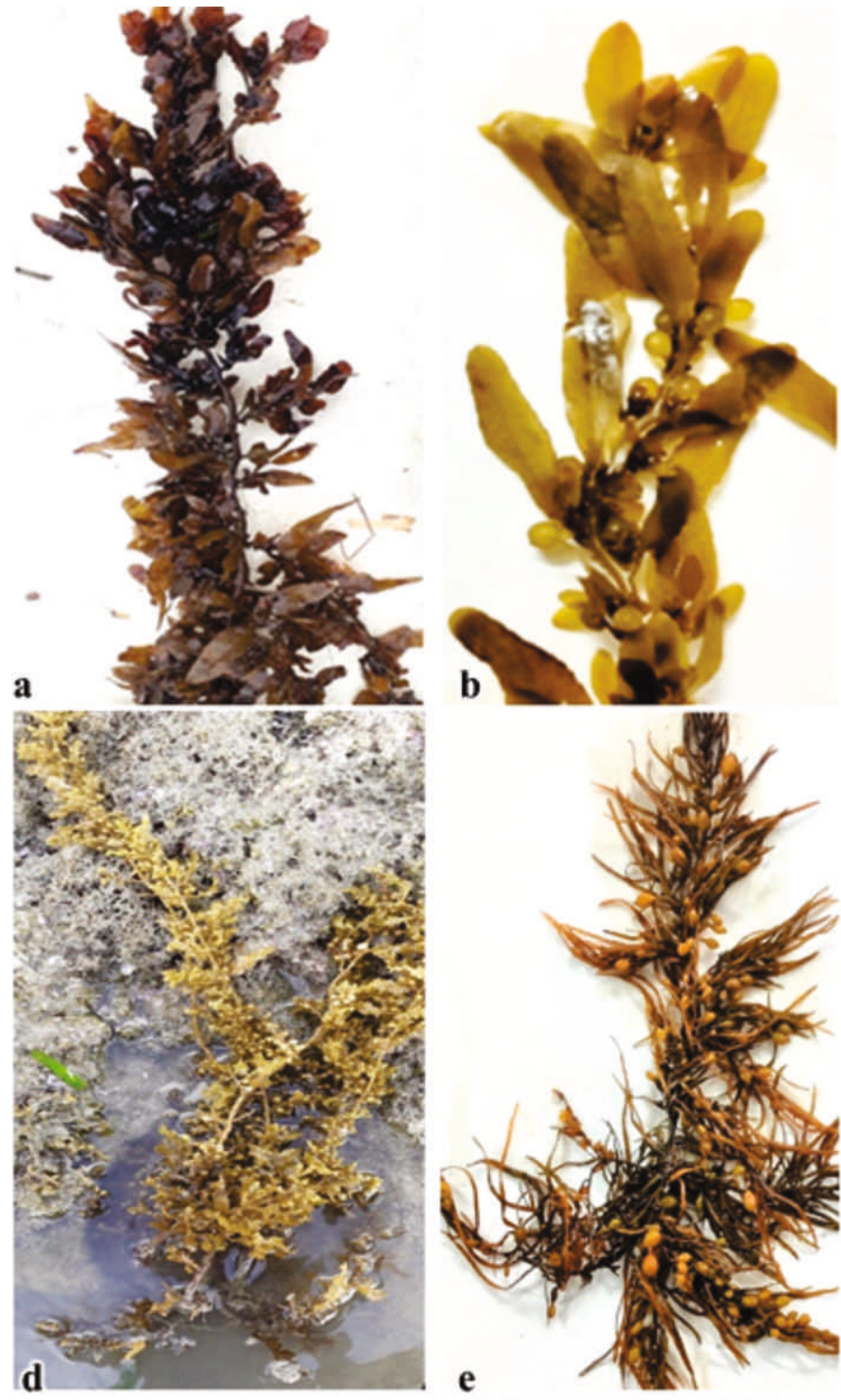

c
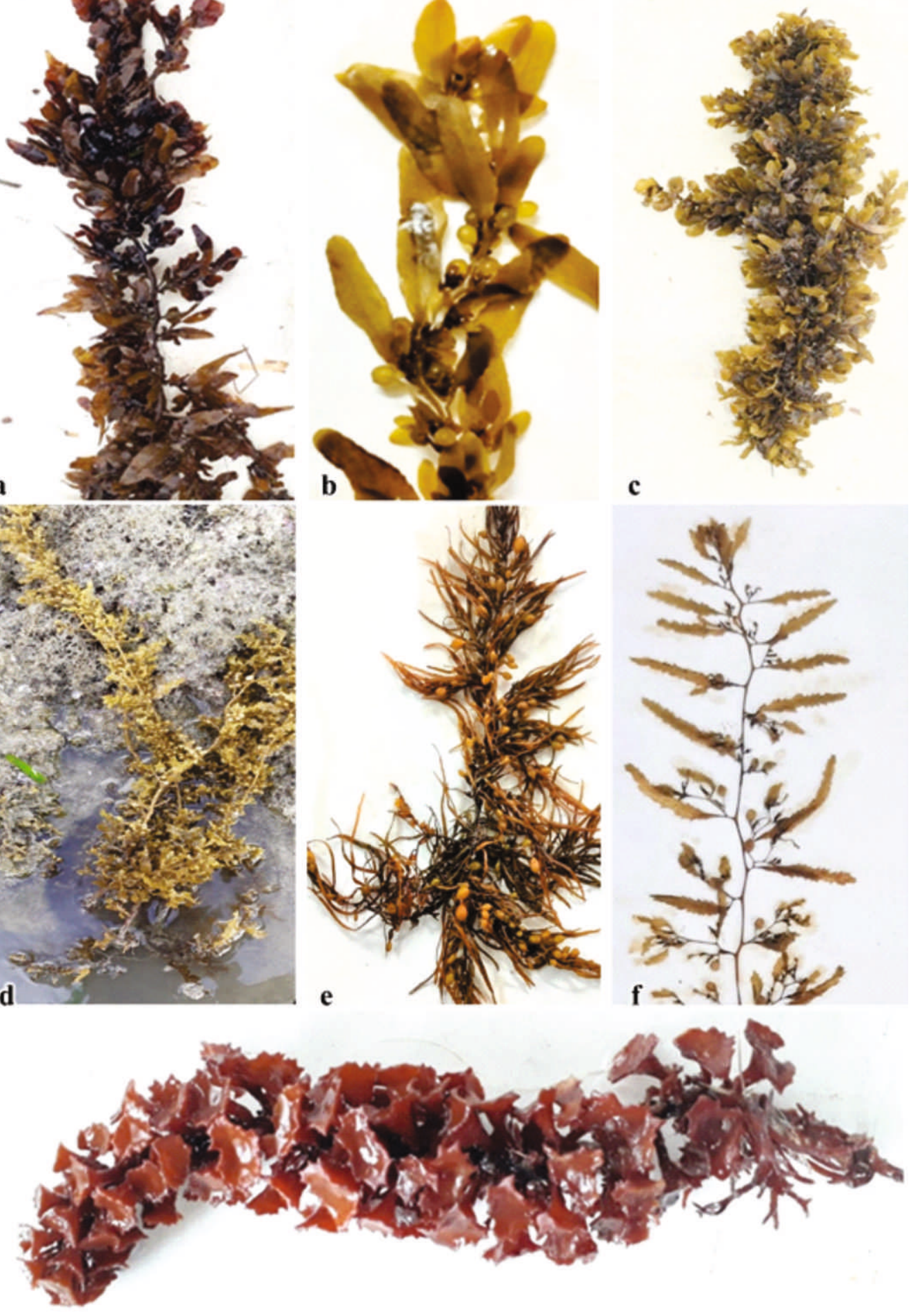

g

Plate 3. Habits of Phaeophyceae: a. Sargassum cinctum; b. Sargassum cinereum; c. Sargassum ilicifolium; d. Sargassum polycystum; e. Sargassum swartzii; f. Sargassum tenerrimum; g. Turbinaria conoides. 
E.C. Oliveira, Alg. Mar. Brasil 217. 1977; Basionym: Dictyota variegata J.V.Lamour.

(Plate 2: j)

Thallus Dark brownish black in color, up to $9 \mathrm{~cm}$ long, crustose, circular to flabellate or rosette, prostrate, tufted, rhizoidal holdfast. Stipe stalked or sessile, rigid. Fronds leafy, fan shaped, irregularly lobed with distinct sub-marginal rows of cells, margins entire. Thallus 46 layered, cells elongated, thin walled, vertically arranged. Generative structures scattered on both sides of fronds.

Specimen examined: Andhra Pradesh: Visakhapatnam District- Bheemunipatnam, 22.03.2017, Palanisamy \& Aron Santhosh Kumar 137158 (MH).

Occurrence: Pre monsoon; Distribution: Gujarat, Kerala and Tamil Nadu.

Padina australis Hauck in Hedwigia 26: 18-21, 44, 1887.

Thallus light to yellowish brown in color, flat, thin, soft, flabellate, attain $7-9 \mathrm{~cm}$ long calcified on the ventral surface. $2-3$ cells thickness, sorus is not covered by indusium. Deeply clefted and divided, concentric rings present over the thallus with regular interval. Generative structures found at the both the surface of the blades.

Specimen examined: Andhra Pradesh: Srikakulam District- Yerramukkam, 20.03.2017, Palanisamy \& Aron Santhosh Kumar 137137 (MH).

Occurrence: Throughout the year; Distribution: Indian Coastline.

Padina boergesenii Allender \& Kraft in Brunonia 6: 87, figs. 6C, H, I, 7C, D. 1983.

(Plate 2: k).

Thallus usually dark brown in color, frondose, flabellate to circular shaped, $4-13 \mathrm{~cm}$ long with rhizomatous holdfast. Fronds leafy, membranous, irregularly cleft into several broad lobes; Thallus 2-4layered, (2-layered in upper portion and 3-4 layered in lower portion). Cells of the upper superficial layer small, rectangular, thin walled, chromatophore content moderate. Reproductive structures form on frond surface.

Specimen examined: Andhra Pradesh: Nellore DistrictPulicat Lake, 27.03.2017, Palanisamy \& Aron Santhosh Kumar 137256 (MH).

Occurrence: Post monsoon; Distribution: Kerala, Gujarat, Goa and Tamil Nadu.

Padina boryana Thivy in W.R. Taylor, Pacific Sci. 20:
355, fig. 2. 1966.

(Plate 2: 1)

Thallus light to dark brown in color, frondose, $4-6 \mathrm{~cm}$ long, lightly calcified, bulbous holdfast. Fronds leafy, spreading, dichotomously branched, surface membranous, irregularly cleft into several lobes, margins entire to slightly undulate. Thallus usually 23 layered, cells of the upper superficial layer small, rectangular, thin walled, chromatophore content more; Reproductive structures develop on frond surface, nonindusiate, tetrasporangia globular to spherical in shape.

Specimen examined: Andhra Pradesh: Nellore DistrictPulicat Lake, 27.03.2017, Palanisamy \& Aron Santhosh Kumar 137340 (MH).

Occurrence: Post monsoon; Distribution: Gujrat, Kerala and Tamil Nadu.

Padina gymnospora (Kuetz.) Sonder in Abh. Naturwiss. Ver Hamburg 5(2): 47. 1871; Basionym: Zonaria gymnospora Kuetz.

(Plate 2: $\mathrm{m}$ )

Thallus light brown in colour, frondose, 7-9 cm, flattened, slightly calcified, Fronds, leafy, membranous, three layers thickness, deeply clefted to forms 3 to 4 lobes, inrolled edge. Cells in surface view, elongated, larger, rectangular. The concentric rings district with regular intervals and alternate on both sides of the blade and the dark lines of spongia. Gametophytes dioecious, antheridia in 1-2 bands in alternate zones.

Specimen examined: Andhra Pradesh: Visakhapatnam District - Behind the Andhra Pradesh Tourism, 24.08.2019, Palanisamy \& Aron Santhosh Kumar $140475(\mathrm{MH})$.

Occurrence: Post monsoon; Distribution: Gujrat \& Tamil Nadu.

Padina pavonica (L.) Thivyin W.R. Taylor, Mar. Alg. East. Trop. Americas: 234. 1960; Basionym: Fucus pavonicus L.

(Plate 2: n)

Thallus olive brown to dark brown in color, frondose, 4-6 cm broad, heavily, tufted, epilithic with bulbous holdfast. Fronds leafy, membranous, dichotomously cleft down, lobes flat, divided into several irregular parts, margins entire to slightly undulate. Thallus 2-4layered, cells small, rectangular or polygonal, chromatophore contents more. Generative assemblies grow mainly near piliferous zones on dorsal surface of frond.

Specimen examined: Andhra Pradesh: Visakhapatnam District- Rushikonda, 24.08.2019, Palanisamy \& Aron Santhosh Kumar 140466 (MH). 
Occurrence: Post monsoon; Distribution: Kerala and Tamil Nadu.

Padina tetrastromatica Hauck in Hedwigia 26: 43. 1887.

(Plate 2: o)

Thallus light yellowish to dark brown in color, frondose, non-calcified, tufted, epilithic. Stipe flat, fronds club shaped, fragile, membranous with alternate rows of microscopic hairs, irregularly cleft into several broad lobes, margins entire, apex obtuse with involute margins. Thallus 4-6 layered, cells small, rectangular, thin walled, chromatophores content more; Reproductive bodies found in transverse rows on thallus surface, tetrasporangia develop in sori on both sides.

Specimen examined: Andhra Pradesh: Vizhianagaram District- Akkupalli, 22.09.2017, Palanisamy \& Aron Santhosh Kumar 137287 (MH).

Occurrence: Post monsoon; Distribution: Andaman \& Nicobar, Karnataka, Kerala, Goa, Gujarat and Tamil Nadu.

Stoechospermum polypodioides (J.V.Lamour.) J. Agardh, Species genera, 100.1848; Basionym: Dictyota polypodioides J.V.Lamour.

(Plate 2: p)

Thallus olive brown to dark brown in colour, up to 26 $\mathrm{cm}$ long and $1-3 \mathrm{~cm}$ broad, frondose, flattened to ribbon shaped, epilithic, rhizomatous hold fast. Fronds foliaceous, thin, tufted, dichotomously branched, glabrous, attenuated base; margins entire to wavy, apical margins undulate or irregularly forked, irregular. Cells in surface view, rectangular, compactly arranged, multi-layered, thick walled, chromatophore content less. Generative bodies scattered on both sides of the thallus.

Specimen examined: Andhra Pradesh: Srikakulam District - Yerramukkam, 22.09.2017, Palanisamy \& Aron Santhosh Kumar 137279 (MH).Occurrence: All seasons; Distribution: Andaman \& Nicobar, Karnataka, Kerala, Goa, Gujarat, Maharashtra and Tamil Nadu.

\section{Order: Fucales; Family: Sargassaceae}

Sargassum cinctum J. Agardh, Spec. Gen. Ord. Alg.1: 324. 1848.

(Plate 3: a)

Plan body dark brown in color, usually up to $40 \mathrm{~cm}$ long, foliose, bushy, tufted, erect, with discoidal holdfast; stipe stalked, simple, terete Fronds well developed, differentiated into primary and secondary branches, glabrous, alternate. Leaves linear- lanceolate $0.8-2.8 \times 0.2-1 \mathrm{~cm}$, thin, transparent or translucent, stalked, base cuneate, surface smooth; midrib prominent and margins serrate. Air bladders and receptacles develop on lateral branches; vesicles oval to spherical, not mucronate.

Specimen examined: Andhra Pradesh: Srikakulam District- Yerramukkam, 20.03.2017, Palanisamy \& Aron Santhosh Kumar 138021 (MH).

Occurrence: All season; Distribution: Karnataka, Kerala, Goa, Gujarat and Tamil Nadu.

Sargassum cinereum J. Agardh, Spec. Gen. Ord. Alg. 1: 305.1848.

(Plate 3: b)

Plant body golden yellow - dark brown in color, usually $26 \mathrm{~cm}$ long, bushy, foliose, tufted, and erect, with discoidal holdfast. Stipe stalked, fronds stout, primary and secondary branches cylindrical. Leaves, simple, wedge shaped, ovate, mature leaves slightly elongate, surface membranous, smooth, transparent, midrib distinct, margins, air bladders and receptacles grow on lateral branches, vesicles spherical, stalked, slightly mucronate and receptacles axillary.

Specimen examined: Andhra Pradesh: Visakhapatnam District- Thotlakonda, 23.03.2017, Palanisamy \& Aron Santhosh Kumar 137218 (MH).

Occurrence: Post monsoon \& Common; Distribution: Karnataka, Kerala, Goa, Gujarat, Maharashtra and Tamil Nadu.

Sargassum ilicifolium (Turner) C.Agardh in Spec. alg. 11.1820; Basionym: Fucus ilicifolius Turner.

(Plate 3: c)

Darkish brown in color, usually $18 \mathrm{~cm}$ long, bushy, foliose, tufted, and erect, with discoidal holdfast. Stipe stalked, fronds stout, distinguished into the primary and secondary branches, cylindrical. Leaves, stalked, simple, wedge shaped or ovate, mature leaves leathery, surface coriaceous, thick, midrib non-distinctive, visible at the base, obtuse apex with serrate or dentate margins, air bladders and receptacles grow on lateral branches, globular, stalked.

Specimen examined: Andhra Pradesh: Visakhapatnam District- Thotlakonda, 26.09.2017, Palanisamy \& Aron Santhosh Kumar 137440 (MH).

Occurrence: Post-monsoon season; Distribution: Andaman \& Nicobar, Kerala, Goa, Gujarat, Maharashtra and Tamil Nadu.

Sargassum polycystum C. Agardh, Syst. Alg.: 304. 1824 .

(Plate 3: d) 
Plant body golden yellow-dark brown in color, $21 \mathrm{~cm}$ long, bushy, tufted, erect with rhizoidal holdfast. Frond bushy, stout, cylindrical to terete, alternately or spirally arranged, densely crowded towards. Leaves simple, small, linear or lanceolate, alternate, thin, membranous, stalked, midrib distinct, margins dentate, vesicles numerous, spherical to oval, receptacles luxuriantly branched.

Specimen examined: Andhra Pradesh: Visakhapatnam District- Thotlakonda, 23.03.2017, Palanisamy \& Aron Santhosh Kumar 137235 (MH).

Occurrence: Post-monsoon season; Distribution: Kerala, Gujarat and Tamil Nadu.

Sargassum swartzii C. Agardh, Syst. Alg.: 1: 120. 1820.

(Plate 3: e)

Dark brown in colour, usually $10-25 \mathrm{~cm}$, tufted, profusely branched, stipe stalked, dark, cylindrical, rough, and stout. Fronds, cylindrical, smooth, densely crowded towards apex. Leaves simple, elongated, linear-lanceolate, alternate, subsessile, thick, coriaceous; midrib distinct, margins wavy and apex acute to obtuse. Vesicles simple, stalked.

Specimen examined: Andhra Pradesh: Visakhapatnam District- Thotlakonda, 10.09.2018 Palanisamy \& Aron Santhosh Kumar 137885 (MH).

Occurrence: Post-monsoon season. Rare \& Distribution: Andaman \& Nicobar, Goa, Gujarat, Maharashtra and Tamil Nadu.

Sargassum tenerrimum J. Agardh, Spec. Gen. Ord. Alg. 1: 305. 1848.

(Plate 3: f)

Plant body yellowish-dark brown in colour, bushy, tufted, erect in young stage, epilithic with discoidal holdfast. Stipe stalked, stout, fronds glabrous, cylindrical. Leaves linear-lanceolate, alternate, thick, coriaceous; midrib prominent, margins sinuate and apex acute. Air bladders and receptacles scattered, vesicles stalked, spherical in shape, antheridia ovoid; oogonia spherical to ovoid.

Specimen examined: Andhra Pradesh: Srikakulam District- Yerramukkam, 22.09.2017, Palanisamy \& Aron Santhosh Kumar 137277 (MH).

Occurrence: Post-monsoon season; Distribution: Andaman \& Nicobar, Karnataka, Kerala, Goa, Gujarat, Maharashtra and Tamil Nadu.

Sargassum vulgare C.Agardh. Species algarum.3.1820.
Plants dark brown to yellowish-brown, up to $30 \mathrm{~cm}$ high, epilithic by conical holdfast. Main axis several, terete, perennial with many primary axes, smooth, alternately or spirally attached. Leaves linearlanceolate, 1.5 to $4 \mathrm{~cm}$ long and 0.3 to $0.7 \mathrm{~cm}$ wide. Crypstostomata inconspicuous or few and scattered, midrib percurrent, asymmetrical and cuneate, margins slightly toothed or dentate. Apex rounded to slightly acute. Vesicles spherical to oblong. Compound receptacle, terete, clusters with warty surface.

Specimen examined: Andhra Pradesh: Visakhapatnam District - Mutyalammapalem, 26.09.2017, Palanisamy \& Aron Santhosh Kumar 137495 (MH).

Occurrence: Post-monsoon season; Distribution: Gujarat and Tamil Nadu.

Sargassum wightii Grev. in J. Agardh, Spec. Gen. Ord. Alg. 1: 329. 1848.

Plant brown in color, $10-22 \mathrm{~cm}$ long, tufted, erect, epilithic, discoidal holdfast. Stipe stalked, cylindrical rough, fronds glabrous; cylindrical or terete, alternately arranged. Leaves narrow, oblong-linear, alternate, stalked, midrib usually inconspicuous; margins entire, apex broadly acute. Vesicles stalked spherical, apex apiculate, conceptacles distinct, both male and female conceptacles develop on separate receptacles.

Specimen examined: Andhra Pradesh: Srikakulam District- Bheemunipatnam, 09.09.2018 Palanisamy \& Aron Santhosh Kumar 137841 (MH).

Occurrence: Post-monsoon season; Distribution: Karnataka, Kerala, Maharashtra and Tamil Nadu.

Turbinaria conoides (J.Agardh) Kuetz. Tabulae phycologicae. 24.1890; Basionym: Turbinaria vulgaris var. conoides J.Agardh.

(Plate 3: g)

Thallus light brown in colour, compact, cone-like, 13 $\mathrm{cm}$ long and radially organized. Firmly attached to substrate by hold fast; main axis and branches slightly compressed. Branchlets turbinate, triangular and dentate concave at the centre and surrounded by fully inner crown of teeth. Vesicles inflated in the middle of the blade.

Specimen examined: Andhra Pradesh: Visakhapatnam District- Behind Andhra Pradesh Tourism, 26.09.2017, Palanisamy \& Aron Santhosh Kumar 137464 (MH).

Occurrence: Post monsoon \& Rare; Distribution: Tamil Nadu. 


\section{ACKNOWLEDGEMENTS}

Authors express their sincere gratitude to Director, BSI, Kolkata and Head of Office, BSI, SRC, Coimbatore for support and encouragements. Also, they thankful to MoEF \& CC, New Delhi for financial assistance under AICOPTAX Scheme.

\section{REFERENCES}

GUNWANT SOKHI AND M.R. VIJAYARAGHAVAN. 1986. Oogonial release in Turbinaria conoides (J. Agardh) Kützing (Fucales, Sargassaceae). Aquatic Botany 24 (4):321-334.

NARASIMHA RAO, G.M., AND SRINIVASA RAO, D. 2016. Seasonal distribution of marine macro algae at Mutyalammapalem, Visakhapatnam district, East Coast of India. J. Pharm. Pharm. Sci.11 (1): 48-51.

PRASANNA LAKSHMI, K. AND G.M.NARASIMHA RAO 2009. Some Numerical studies on Marine algae of Visakhapatnam Coast. J. Algal Biomass Utln. 1 (1); $60-85$.

RAO, G.M. NARASIMHA 1992. Seasonality in growth, reproduction and spore liberation of Porphyra vietnamensis Tanaka et Ho (Rhodophyta, Bangiales). Mahasagar. A Journal of Limnology and Oceanography 25: 11-17.

RAO, G.M. NARASIMHA AND M. UMAMAHESWARA RAO 1986. Numerical study of intertidal zonation along Visakhapatnam coast, east coast of India. Indian J. Mar. Sci. 15: 102-106.

RAO, G.M. NARASIMHA, G. SUBBA RANGAIAH AND S.V.V.S.N. DORA 2008. Spore shedding in Catenella impudica from the Godavari Estuary at Bhiravapalem, India. Algae 23(1):71-74.
RAO, M. UMAMAHESWARA AND SREERAMULU 1964. An ecological study of some intertidal algae of the Visakhapatnam coast. Journal of Ecology 52: 595616.

RAO, M. UMAMAHESWARA AND T. SREERAMULU, 1968. Recolonization of algae on denuded rocky surface of the Visakhapatnam coast. Bot. Mar. 11: 122-126.

RAO, M. UMAMAHESWARA AND T. SREERAMULU, 1970a. An annotated list of the marine algae of Visakhapatnam (India). Bot. J. Linn. Soc. 63: 23-45.

RAO, P.S.N. AND R.K. GUPTA 2015. Algae of India, Vol 3: A checklist of Indian Marine Algae (Excluding Diatoms \& Dinoflagellates). Botanical Survey of India, Kolkata.

ROTT E. AND WEHR J.D. 2016 The Spatio-Temporal Development of Macroalgae in Rivers. In: Necchi JR O. (Ed.) River Algae. Springer, Cham, 159-195.

SATYA RAO, K., PRAYAGA MURTY, P., AND NARASIMHA RAO, G.M. 2011. Seasonal Studies on Marine algae of the Bhimili Coast, East Coast of India. J. Algal Biomass Utln. 2 (2): 69-82.

SREERAMULU.1952. On a Porphyra from Waltair Coast. Sci. Cult. 18: 285-286.

SREERAMULU. 1953. A Codium from the Coromandel Coast. J. Indian Bot. Soc. 32: 67-69.

VISHNUPRIYA SOWJANYA, I., AND RAJA SEKHAR, P.S. 2015. A comparative study of seaweed flora over a period of time with reference to climate change in Visakhapatnam Coast, Andhra Pradesh, India. Adv. Appl. Sci. Res. 6(6):187-193

VISHNUPRIYA SOWJANYA, I., AND RAJA SEKHAR, P.S. 2017. Ecology of marine macro algal flora of Visakhapatnam coastal areas, Bay of Bengal, India. J. Threat. Taxa 9(3): 9911-9919. 